\title{
Especificidad de forófito y preferencias microambientales de los líquenes cortícolas en cinco forófitos del bosque premontano de finca Zíngara, Cali, Colombia
}

\author{
Edier Soto Medina ${ }^{1}$, Robert Lücking ${ }^{2} \&$ Ana Bolaños Rojas ${ }^{1}$ \\ 1. Universidad del Valle, Facultad de Ciencias Naturales, Departamento de Biología, Cali, Colombia, Calle 13 \\ N.100-00; crisbol123@gmail.com, ediersot@gmail.com \\ 2. Departamento de Botánica, The Field Museum, Chicago, IL 60605, USA; rlucking@ fieldmuseum.org
}

Recibido 17-V-2011. Corregido 15-IX-2011. Aceptado 18-X-2011.

\begin{abstract}
Phorophyte specificity and microenvironmental preferences of corticolous lichens in five phorophyte species from premontane forest of Finca Zíngara, Cali, Colombia). Lichenized fungi or lichens are organisms that have been little studied in the tropics and which distribution is affected by microenvironmental factors and substrate characteristics. The present study aimed to identify phorophyte specificity and microenvironmental preferences of corticolous lichens in five phorophyte species from premontane forest of the farm Finca Zingara in Cali, Colombia. For this, five individuals were selected from five tree species (phorophytes). Lichen species present in a $0.50 \times 0.20 \mathrm{~m}^{2}$ quadrant located in the trunk of each tree at a height of $1.3 \mathrm{~m}$ were identified. Substrate parameters such as bark pH, Diameter Breast Height (DBH) and bark structure were measured. Also, microenvironmental factors including temperature, humidity and irradiance were determined. In order to detect phorophyte preferences, a non-metric multidimensional scaling (NMS) and indicator species analysis were made. Spearman correlation analysis was used to assert the relationship between environmental variables and groupings found in the NMS. A total of 69 species of lichens were found, of which 37 were identified to species, 18 to genera and 14 were not determined because they were sterile or had no spores. NMS showed that some individuals of the same tree species were grouped alongside the analysis dimensions, and they were related with the factors of light intensity, temperature and DBH. Only three lichens with preference for certain tree species were found (Arthonia microsperma by Meriania sp., Cladonia ceratophylla and sorediado 8 by Clusia sp.), suggesting absence of phorophyte preferences. Thus, it can be concluded that lichens from the study area do not show phorophyte preference, but their distribution is affected by light, temperature and DBH. Rev. Biol. Trop. 60 (2): 843-856. Epub 2012 June 01.
\end{abstract}

Key words: corticolous lichens, phorophyte specificity, microenvironmental factors, non-metric multidimensional scaling (NMS), correlation, indicator species analysis.

Los bosques montanos son uno de los ecosistemas más amenazados en Colombia, debido a la deforestación, el aumento de la agricultura, el pastoreo, las quemas y la sobreexplotación de sus recursos; esta situación se incrementa cada vez más con el aumento de la población, que gran parte se ubica en la región andina (Armenteras et al. 2007). En estos bosques, las especies epífitas constituyen uno de los principales componentes de la diversidad vegetal (Gentry \& Dodson 1987), donde adquieren gran importancia en el balance hídrico y en el ciclo de nutrientes (Holz 2003). En Colombia, son escasos los estudios sobre líquenes epífitos, y dentro de ellos, apenas se han tenido en cuenta a las especies crustáceas (microlíquenes), que son los que más contribuyen a la diversidad de estos organismos en el trópico (Wolf 1993, Holz 2003, Lücking et al. 2009).

Los líquenes epífitos son particularmente sensibles a los cambios climáticos (Nash \& Olafsen 1995, Rivas-Plata et al. 2008); son organismos que carecen de mecanismos que regulen la captación y pérdida de agua y luz 
(Nash 1996, Renhorn et al. 1997); además, adquieren el agua y la mayor parte de los nutrientes directamente de la atmósfera, por lo que, presentan un contenido de agua semejante al del medio en el que crecen (Selva 1996, McCune 2000). Al no poder seleccionar las sustancias que absorben, sus talos quedan en estrecha relación con el ambiente (Selva 1996, McCune 2000). Los líquenes están fuertemente influenciados tanto por variables macro como microambientales, que afectan a su riqueza, abundancia y distribución a diferentes escalas (McCune et al. 1997). A nivel regional, son las variables orográficas y climáticas las que condicionan la composición de especies (Hauck \& Spribille 2005, Werth et al. 2005), mientras que a nivel local, son las variables microclimáticas, relacionadas con la estructura del bosque como la edad y el tipo de árbol o la cobertura del dosel, las que van a condicionar las comunidades (Belinchón et al. 2009, Aragón et al. 2010).

Pese a que se han realizado pocos estudios en el trópico en lo concerniente a la preferencia de forófito de los líquenes (Sipman \& Harris 1989, Gradstein 1992, Wolf 1993, Gradstein et al. 1996, Merwin \& Nadkarni 2001, Coppins \& Wolseley 2002, Cáceres et al. 2007, Rosabal et al. 2010), se ha encontrado que estos organismos muestran patrones similares a los de zonas templadas (Sipman \& Harris 1989, Boonpragob \& Polyiam 2007). Por ejemplo, en dos bosques de Guyana, los líquenes presentaron una ligera especificidad de forófito y una pronunciada zonación vertical desde la vegetación baja de sombra hasta el dosel de los árboles (Cornelissen \& ter Steege 1989, Montfoort \& Ek 1990). Wolf (1993), en diferentes tipos de bosque en Colombia, observó correlaciones de formación de comunidades liquénicas con parámetros del sustrato (tales como tipo de corteza, pH) y contenido de nutrientes. En Costa Rica, Holz (2003) reportó para dos especies de Quercus, que el gradiente vertical (desde la base hasta las ramas de los árboles) de luz y humedad relativa, es el factor más importante en la diferenciación de las comunidades de criptógamas epífitas (líquenes y briófitos). En este estudio algunas especies también tuvieron preferencias por una de las dos especies de Quercus, lo cual podría ser explicado por diferencias en el $\mathrm{pH}$, retención de agua y dureza de la corteza. También se ha encontrado en estudios con líquenes folícolas, que el microclima es el factor más determinante en la formación de comunidades, mientras que las preferencias de forófito son ausentes o sutiles (Nowak \& Winkler 1975, Barillas et al. 1993, Lücking 1999, Nöske 2004, Nöske \& Sipman 2004, Holz \& Gradstein 2005).

Marcelli (1992) fue el primero en investigar microlíquenes en los manglares del sureste de Brasil, y encontró que las comunidades de líquenes responden a parámetros microclimáticos (luz, humedad) y también se correlacionan con características de la corteza del árbol. Komposch \& Hafellner (1999, 2000, 2002, 2003), en un bosque húmedo de la parte superior del río Orinoco en Venezuela, confirmaron la existencia de diferentes comunidades de líquenes a diferentes alturas de los árboles. Por otro lado, Cáceres et al. (2007) en un bosque húmedo tropical en el noroeste de Brasil, observaron que la formación de comunidades de líquenes no solamente está determinada por las características de la corteza y por parámetros microclimáticos, sino, en gran parte, por el efecto estocástico de la dispersión de las especies.

El objetivo de este trabajo fue evaluar la especificidad de forófito y las preferencias microambientales de los líquenes cortícolas en el Bosque de la Finca Zíngara, Cali, Colombia. Las variables ambientales usadas fueron la humedad relativa, la temperatura, la intensidad de luz, el pH del sustrato y la estructura de la corteza.

\section{MATERIALES Y MÉTODOS}

Zona de estudio: El estudio se realizó en la finca Zíngara (coordenadas 332' N-76³6' W: entre los 1900-2000msnm), ubicada en el corregimiento de La Elvira, Municipio de Cali, a $4 \mathrm{~km}$ de la vereda $\mathrm{Km} 18$ (en la carretera que lleva desde éste corregimiento hasta Dapa), Colombia. Esta finca es propiedad de la familia Giraldo-Gensini y presenta un buen estado de 
conservación, razón por la cual se han realizado varias investigaciones botánicas y zoológicas (Gentry \& Dodson 1987, Giraldo 1988). La zona de estudio presenta un patrón bimodal de lluvias, con un pico en abril-mayo y el otro en octubre-noviembre. El promedio anual de precipitación es de $1647 \mathrm{~mm}$; la temperatura media es de $16^{\circ} \mathrm{C}$ y fluctúa entre los $12^{\circ} \mathrm{C}-20^{\circ} \mathrm{C}$ (Giraldo 1988). La finca está situada en la vertiente oriental de la Cordillera Occidental de Los Andes de Colombia dentro de la formación de bosque muy húmedo montano bajo (bmhMB). La vegetación está constituida principalmente por bosque de niebla, en el cual hay algunas zonas de bosque primario en la parte más alta y bosques de transición. El bosque presenta una pendiente de aproximadamente 30-60 (Giraldo 1988).

Muestreo: Asimismo, se seleccionaron al azar cinco individuos de cinco especies de árboles (Clusia sp., Heliocarpus americanus L., Meriania sp., Saurauia brachybotrys Turcz. y Schefflera ferruginea (Willd. ex Schult.) Harms) a lo largo de un camino de $100 \mathrm{~m}$, el cual cruza el bosque en sentido norte-sur. Las recolectas se realizaron en los meses de junio y julio del 2009, en cuatro salidas de campo. En cada forófito se ubicó un cuadrante de $0.50 \times 0.20 \mathrm{~m}$ a $1.3 \mathrm{~m}$ de altura desde la base y en el lado oriental del tronco. Los hongos liquenizados recolectados se colocaron en bolsas de papel para su posterior identificación en el laboratorio. También, se estimó la abundancia relativa (porcentaje de cobertura) de cada especie en el cuadrante. Adicionalmente, en cada unidad de muestreo se midió la humedad relativa, la temperatura ambiente (higrotermómetro digital) e irradianza (cuantómetro LI-1 000 DataLogger LI-COR), con dos mediciones en el día (una en la mañana y otra en la tarde). Además, se registró el DAP (diámetro a la altura del pecho), el pH de la corteza y la estructura de la corteza (Cáceres et al. 2007) (Cuadro 1). La estructura de la corteza fue estudiada en términos de la presencia de lenticelas, mediante el número de lenticelas por $\mathrm{cm}^{2}$ y el tamaño medio de éstas.
Dado que a lo largo del camino las condiciones ambientales van a ser diferentes, se procedió a agrupar los árboles tal y como muestra la figura 1. De esta forma se podrá observar si los líquenes presentan una zonación en su distribución. El grupo 1 comprendió a los árboles Clusia 1, 2, 3 y 4, Heliocarpus 2, Meriania 1 y Schefflera 5; el grupo 2 a los forófitos Clusia 5, Heliocarpus 1 y 3, Meriania 2, 3, 4 y 5, Saurauia 5 y Schefflera 1; y el grupo 3 estuvo conformado por Heliocarpus 4 y 5, Saurauia 1, 2, 3 y 4, Schefflera 2, 3 y 4 (Fig. 1).

Para la identificación de los especímenes, se siguieron las claves de líquenes neotropicales de Sipman (2006) y del género Coenogonium de Rivas-Plata et al. (2006). Los especímenes se depositaron en el herbario de la Universidad del Valle (CUVC).

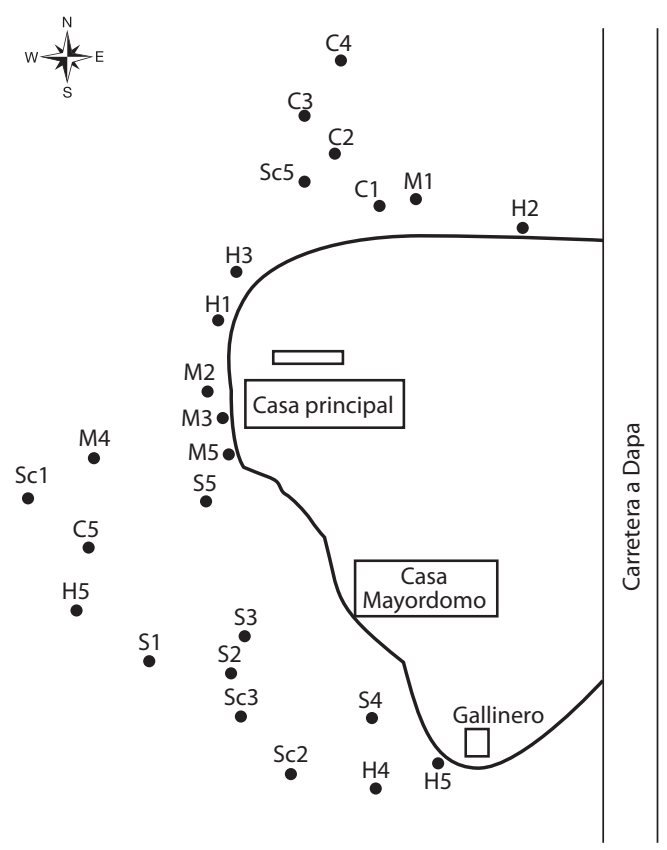

Fig. 1. Mapa del camino en el que se muestrearon los líquenes de los 25 forófitos del estudio. (C) indica Clusia, (H) Heliocarpus, (M) Meriania, (S) Saurauia y (Sc) Schefflera.

Fig. 1. Map of the way where the lichens from the 25 phorophytes studied weresampled. (C) indica Clusia, (H) Heliocarpus, (M) Meriania, (S) Saurauia and (Sc) Schefflera. 
CUADRO 1

Riqueza (S) y parámetros microambientales

TABLE 1

Richness (S) and microenvironmental parameters

\begin{tabular}{|c|c|c|c|c|c|c|}
\hline Forófito & S & $\mathrm{pH}$ & $\mathrm{DAP}(\mathrm{m})$ & Intensidad de luz $\left(\mathrm{W} / \mathrm{m}^{2}\right)$ & Humedad relativa & Temperatura $\left({ }^{\circ} \mathrm{C}\right)$ \\
\hline Clusia 1 & 8 & 5.54 & 0.150 & 24.50 & 94 & 21 \\
\hline Clusia 2 & 7 & 5.89 & 0.796 & 24.70 & 89 & 20 \\
\hline Clusia 3 & 5 & 5.38 & 0.939 & 26.60 & 89 & 20 \\
\hline Clusia 4 & 7 & 5.58 & 0.121 & 11.85 & 89.5 & 21 \\
\hline Clusia 5 & 6 & 5.5 & 0.828 & 10.70 & 89 & 21.5 \\
\hline Promedio \pm SD & 7 & $5.58 \pm 0.19$ & $0.105 \pm 0.296$ & $19.67 \pm 7.71$ & $90.1 \pm 2.19$ & $20.7 \pm 0.67$ \\
\hline Heliocarpus 1 & 2 & 6.61 & 0.416 & 24.70 & 86 & 21 \\
\hline Heliocarpus 2 & 6 & 6.64 & 0.505 & 53.95 & 92 & 21.5 \\
\hline Heliocarpus 3 & 3 & 7.58 & 0.436 & 29.50 & 98 & 19 \\
\hline Heliocarpus 4 & 5 & 6.51 & 0.226 & 42.50 & 87.5 & 21 \\
\hline Heliocarpus 5 americanus 5 & 6 & 7.22 & 0.252 & 53.85 & 96 & 20.5 \\
\hline Promedio \pm SD & 4 & $6.91 \pm 0.47$ & $0.367 \pm 0.123$ & $40.9 \pm 13.54$ & $91.9 \pm 5.2$ & $20.6 \pm 0.96$ \\
\hline Meriania 1 & 5 & 4.84 & 0.194 & 16.635 & 95 & 20 \\
\hline Meriania 2 & 5 & 4.72 & 0.411 & 27.2 & 86 & 21 \\
\hline Meriania 3 & 3 & 5.45 & 0.32 .8 & 14.4 & 90.5 & 22 \\
\hline Meriania 4 & 5 & 4.47 & 0.414 & 21.85 & 90.5 & 17.5 \\
\hline Meriania 5 & 4 & 5.28 & 0.331 & 25.6 & 92.5 & 21.5 \\
\hline Promedio \pm SD & 7 & $4.95 \pm 0.40$ & $0.336 \pm 0.892$ & $21.14 \pm 5.54$ & $90.9 \pm 3.03$ & $20.4 \pm 1.78$ \\
\hline Saurauia 1 & 6 & 6.55 & 0.164 & 25.4 & 86 & 23 \\
\hline Saurauia 2 & 6 & 7.15 & 0.193 & 33.1 & 91.5 & 20.5 \\
\hline Saurauia 3 & 11 & 6.95 & 0.147 & 33.2 & 93 & 19 \\
\hline Saurauia 4 & 11 & 6.85 & 0.169 & 33.8 & 95.5 & 19.5 \\
\hline Saurauia 5 & 4 & 6.88 & 0.143 & 36.35 & 93.5 & 19.5 \\
\hline Promedio \pm SD & 8 & $6.88 \pm 0.22$ & $0.163 \pm 0.198$ & $32.37 \pm 4.11$ & $91.9 \pm 3.60$ & $20.3 \pm 1.60$ \\
\hline Schefflera 1 ferruginea 1 & 5 & 6.95 & 0.955 & 20.65 & 88 & 23 \\
\hline Schefflera 2 & 5 & 7.8 & 0.142 & 31.4 & 87 & 17.5 \\
\hline Schefflera 3 & 3 & 6.25 & 0.129 & 30 & 91 & 20.5 \\
\hline Schefflera 4 & 3 & 7.22 & 0.955 & 32.75 & 85 & 19.5 \\
\hline Schefflera 5 & 4 & 6.53 & 0.924 & 17.765 & 91.5 & 20.5 \\
\hline Promedio \pm SD & 4 & $6.95 \pm 0.60$ & $0.111 \pm 0.229$ & $26.51 \pm 6.82$ & $88.5 \pm 2.74$ & $20.2 \pm 2.0$ \\
\hline
\end{tabular}

Ahora bien, se empleó estadística descriptiva para resumir y mostrar los patrones de los datos registrados, tanto de las especies encontradas como de los parámetros ambientales y de sustrato medidos. Las variables de respuesta utilizadas fueron la riqueza de especies por forófito o diversidad alfa y la diversidad beta (recambio de especies entre forófitos) (McCune et al. 2002). La diversidad beta fue medida con el índice de disimilitud de Sørensen (McCune et al. 2002). También, se efectuó una curva de acumulación de especies para evaluar la eficiencia del muestreo (McCune et al. 2002). Las comparaciones se evaluaron con pruebas de Kruskal-Wallis al 95\% de confianza.

Por consiguiente, se usó un procedimiento de permutación de respuesta múltiple (MRPP) para comparar la composición de especies entre forófitos y entre los tres grupos. Este procedimiento no paramétrico permitió evaluar la hipótesis nula de no diferencias significativas en la composición de especies entre grupos previamente definidos. Para este fin, el método usa una prueba $\mathrm{T}$ para establecer si hay 
diferencias entre los grupos. Se utilizó el índice de disimilitud de Sørensen para el MRPP (McCune et al. 2002).

Para evaluar la preferencia de forófito, los datos se analizaron mediante un escalamiento no métrico multidimensional (NMS) en donde se usó el índice de disimilitud de Sørensen, con el fin hallar asociaciones entre muestras (forófitos) basadas en la composición de especies de líquenes. El análisis se corrió con 100 iteraciones en total, para evaluar la estabilidad se uso un criterio de 0.05 y 20 iteraciones. Además, se realizó un análisis de correlación lineal de Spearman entre las dos primeras dimensiones obtenidas en el NMS y los parámetros que se midieron en cada forófito, para evaluar si los agrupamientos que se encontraron en NMS estaban relacionados con estas variables (McCune et al. 2002). También se efectuó un análisis de especies indicadoras (simulación de Monte Carlo) para identificar las especies de líquenes que son específicas para determinadas especies de forófitos. Igualmente, se usaron 4999 aleatorizaciones en la simulación de Monte Carlo. Este análisis también se usó para ver si había especies indicadoras para los tres grupos de árboles. Los análisis estadísticos fueron efectuados con el software STATISTICA® 7.0 (Statsoft 2004) y PC-ORD® 5.0 (McCune et al. 2002).

\section{RESULTADOS}

Parámetros microclimáticos y de micrositio: La intensidad de luz fue mayor para los individuos de Heliocarpus americanus (promedio $40.9 \mathrm{~W} / \mathrm{m}^{2}$ ) y baja para los de Clusia $\left(19.67 \mathrm{~W} / \mathrm{m}^{2}\right)$ (Cuadro 1). En contraste con los parámetros anteriores, la temperatura ambiental y la humedad relativa, no variaron demasiado entre las especies de forófitos. $\mathrm{El} \mathrm{pH}$ de la corteza fue más bajo para los individuos de Meriania sp. (promedio de 4.95) y más alto para los de Schefflera ferruginea (promedio de 6.95) (Cuadro 1). El DAP fue más bajo para los individuos de Clusia sp. $(0.1054 \mathrm{~m})$ y para los individuos de Heliocarpus americanus el más alto $(0.3669 \mathrm{~m})$ (Cuadro 1$)$.
Al comparar los tres grupos formados para los parámetros microambientales, se encontró que solamente las variables de $\mathrm{pH}$ e intensidad de luz presentaron diferencias significativas (Kruskal-Wallis, p<0.05) (Fig. 2). Los grupos 1 y 2 no presentan diferencias significativas para las variables microclimáticas y de micrositio (comparación por parejas de Mann-Whitney, $\mathrm{p}>0.05$ ), mientras que estos dos grupos si difieren significativamente en las condiciones de luz y pH de la corteza del grupo 3 (Fig. 2). De acuerdo con lo anterior, se tiene que los árboles del grupo 3 presentaron una mayor irradianza y una corteza con mayor $\mathrm{pH}$ en comparación a los grupos 1 y 2 (Fig. 2).

Diversidad de especies de líquenes: $\mathrm{Se}$ encontraron 69 especies de líquenes en los 25 forófitos estudiados, de los cuales 37 fueron determinados hasta especie, 18 hasta género y 14 no fueron determinados debido a que estaban estériles. Se hallaron 27 géneros agrupados en 16 familias (Cuadro 2). Los géneros con el mayor número de especies fueron Herpothallon (12), Pyrenula (4), Graphis (4), Arthonia, Coenogonium y Phaeographis (3) (Cuadro 2). La curva de acumulación de especies muestra que la riqueza de especies aumenta progresivamente con el número de unidades de muestreo y la curva no tiende a estabilizarse (Fig. 3). El estimador de Jackknife calculó una riqueza de 106 especies de líquenes, lo que indica que el muestreo tuvo una eficiencia del $65 \%$.

Los individuos 3 y 4 de Saurauia brachybotrys presentaron la mayor riqueza o diversidad alfa de líquenes (11 especies), mientras que el individuo 1 de Heliocarpus americanus mostró la menor riqueza (2 especies) (Cuadro 1). De igual forma, los árboles de S. brachybotrys presentaron la mayor diversidad alfa promedio (8 especies), seguido de los de Clusia sp. (7 especies), mientras que en las otras tres especies de forófitos se encontraron 4 especies de líquenes en promedio (Cuadro 1). El 76\% de los forófitos presentaron entre 3 y 6 especies de líquenes (Fig. 4). El 78\% de las especies (54) fueron raras, 39 de las cuales se encontraron en un sólo forófito y 15 en dos forófitos (Fig. 4). 

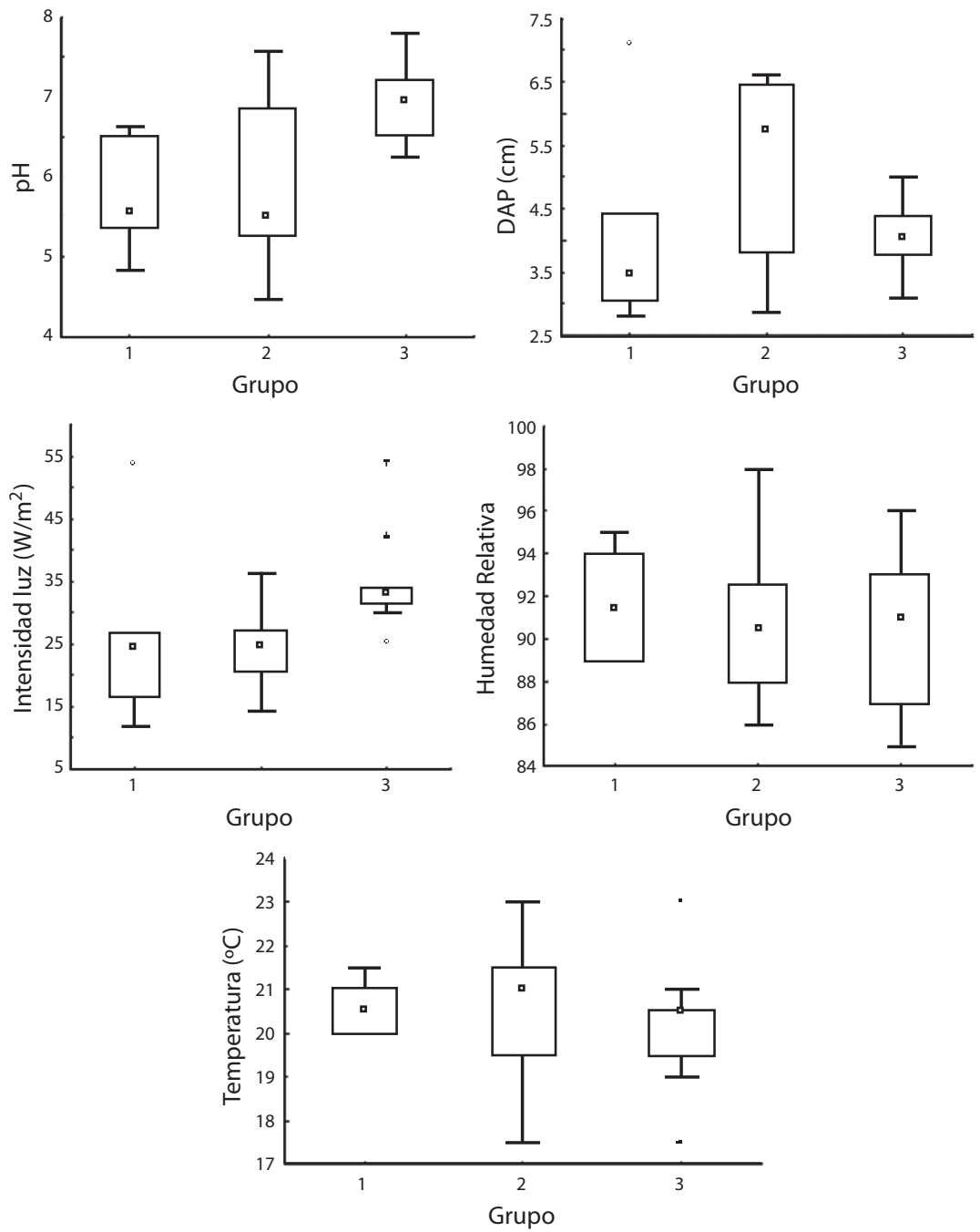

Fig. 2. Diagramas de caja para los parámetros microclimáticos y de micrositio para los tres grupos formados a lo largo del camino. En el gráfico se muestra la mediana en la parte central de la caja y el rango intercuartil.

Fig. 2. Box plots for microclimatic and microsite parameters for the three groups formed along the way. The graph shows the median in the middle of the box and interquartile range.

Por otro lado, la especie sorediado 7 presentó la mayor frecuencia (9 de los 25 forófitos), seguido por sorediado 4 con 6 y Cladonia ceratophylla y Herpothallon sp.2. El grupo 1 y el grupo 3, presentaron la mayor riqueza de líquenes ( 6 especies en promedio), mientras que el grupo 2 sólo presentó 4 especies de líquenes en promedio (Cuadro 1).
Diversidad beta: Del total de comparaciones por pareja para la diversidad beta, medida como el índice de disimilitud de Sørensen, el $91 \%$ estuvieron por encima de 0.7 (Fig. 5A), lo cual indica una alta diversidad beta o heterogeneidad entre forófitos. La diversidad beta entre forófitos de la misma especie fue más baja frente a árboles de diferentes 
CUADRO 2

Características de las especies de líquenes encontradas en los forófitos

TABLE 2

Traits of the lichen species found in the sampled phorophytes

\begin{tabular}{|c|c|}
\hline Especie & Familia \\
\hline Architrypethelium hyalinum Aptroot & Trypetheliaceae \\
\hline Arthonia aff. microsperma Nyl. & Arthoniaceae \\
\hline Arthonia cf. complanata Fée & Arthoniaceae \\
\hline Arthonia sp. & Arthoniaceae \\
\hline Auriculora byssomorpha (Nyl.) Kalb & incertae sedis \\
\hline Bacidina sp. & Ramalinaceae \\
\hline Byssoloma chlorinum (Vain.) Zahlbr. & Pilocarpaceae \\
\hline Chapsa pseudoschizostoma (Hale) INED. & Graphidaceae \\
\hline Cladonia ceratophylla (Sw.) Spreng. & Cladoniaceae \\
\hline Coenogonium geralense (Henn.) Lücking & Coenogoniaceae \\
\hline Coenogonium linkii Fée & Coenogoniaceae \\
\hline Coenogonium subdentatum (Vĕzda \& G. Thor) Rivas Plata, Lücking, Umaña \& Chaves & Coenogoniaceae \\
\hline Dichosporidium nigrocinctum (Ehrenb.) G. Thor & Roccelaceae \\
\hline Diorygma minisporum Kalb, Staiger \& Elix & Graphidaceae \\
\hline Eschatogonia prolifera (Mont.) R. Sant. & incertae sedis \\
\hline Everniastrum limaeforme (Taylor) Hale ex Sipman & Parmeliaceae \\
\hline Graphis adpressa Vain. & Graphidaceae \\
\hline Graphis sp. 1 & Graphidaceae \\
\hline Graphis sp. 2 & Graphidaceae \\
\hline Graphis tumidula (Fée) Spreng. & Graphidaceae \\
\hline Hemigrapha asteriscus (Müll. Arg.) D. Hawksw. & Parmulariaceae \\
\hline Hemithecium cf.laubertianum (Fée) Staiger & Graphidaceae \\
\hline Herpothallon globosum $\mathrm{G}$. Thor & Arthoniaceae \\
\hline Herpothallon rubrocinctum (Ehrenb.) Aptroot, Lücking \& G. Thor & Arthoniaceae \\
\hline Hypotrachyna sp. & Parmeliaceae \\
\hline Laurera effusa Aptroot \& Sipman & Trypetheliaceae \\
\hline Leptogium laceroides (B. de Lesd.) P. M. Jørg. & Collemataceae \\
\hline Ocellularia clandestina (Ach.) Müll.Arg. 1 & Graphidaceae \\
\hline Ocellularia clandestina (Ach.) Müll.Arg. 2 & Graphidaceae \\
\hline Parmotrema sp. & Parmeliaceae \\
\hline Phaeographis brasiliensis (A. Massal.) Kalb and Matthes Leicht & Graphidaceae \\
\hline Phaeographis scalpturata (Ach.) Staiger & Graphidaceae \\
\hline Phaeographis spondaicum (Nyl.) Staiger & Graphidaceae \\
\hline Porina guaranitica Malme & Porinaceae \\
\hline Pseudocyphellaria aurata (Ach.) Vain. & Lobariaceae \\
\hline Pyrenula concatervans (Nyl.) R. C. Harris & Pyrenulaceae \\
\hline Pyrenula macrocarpa A. Massal. & Pyrenulaceae \\
\hline Pyrenula tenuisepta R. C. Harris & Pyrenulaceae \\
\hline Pyrenula mamillana (Ach.) Trevisan & Pyrenulaceae \\
\hline Redingeria leiostoma (Tuck.) Frisch & Graphidaceae \\
\hline Sticta sp. 1 & Lobariaceae \\
\hline Sticta sp. 2 & Lobariaceae \\
\hline Strigula phaea (Ach.) R.C. Harris & Strigulaceae \\
\hline Thelotrema adjetum Nyl. & Graphidaceae \\
\hline Thelotrema leucomelaenum Nyl. & Graphidaceae \\
\hline
\end{tabular}




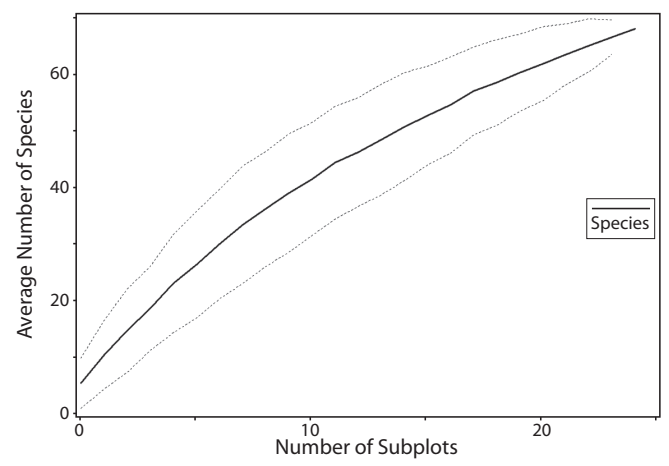

Fig. 3. Curva de acumulación de especies para las 25 unidades de muestreo.

Fig. 3. Species accumulation curve for the 25 sampling units.
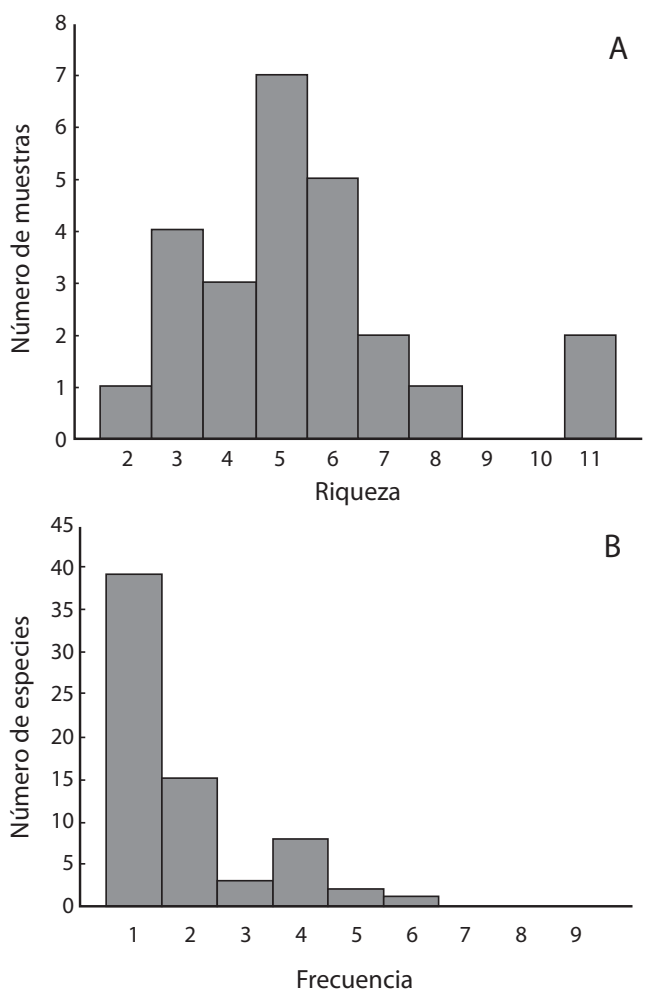

Fig. 4. Riqueza de especies en todas las muestras (A) y la frecuencia (B). Nótese que el $76 \%$ de los forófitos presentaron entre 3 y 6 especies de líquenes (A) y el alto porcentaje de especies raras (B).

Fig. 4. Species richness behaviour in all samples (A) and the frequency (B). Note that $76 \%$ of phorophytes occurred between 3 and 6 species of lichens (A) and the high percentage of rare species (B).
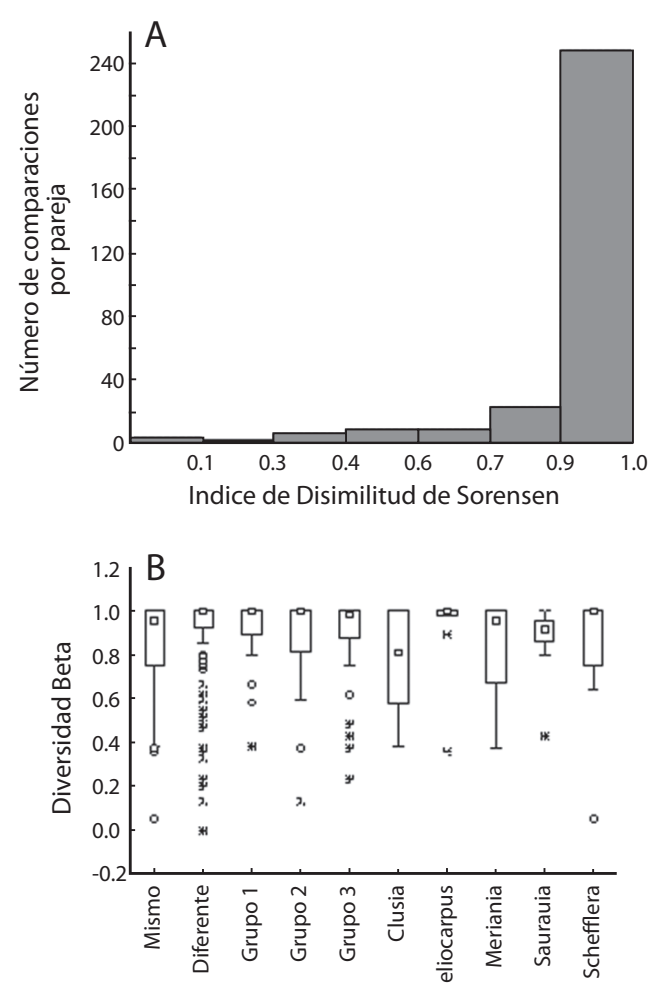

Fig. 5. Diversidad beta (índice de disimilitud de Sørensen) para las 300 parejas formadas (A) y Diagrama de cajas para diversidad beta (índice de disimilitud de Sørensen) para los grupos de forófitos (B).

Fig. 5. Beta diversity (Sørensen similarity index) for the 300 couples formed (A) and beta diversity box-chart (Sørensen dissimilarity index) for phorophyte groups.

especies (1.00 misma vs. 0.957 diferente). No se encontraron diferencias significativas para la diversidad beta entre las cinco especies de forófitos (Kruskal-Wallis, p=0.324) (Fig. 5B). Sin embargo, se observó que para Clusia sp. fue más baja la mediana del índice de disimilitud de Sørensen (0.77) y más alta para Heliocarpus americanus (0.92) (Fig. 5B). La prueba de Kruskal-Wallis para comparar la diversidad beta de los tres grupos sugiere que no hay diferencias significativas entre estos $(\mathrm{p}=0.84)$.

MRPP, NMS y análisis de especies indicadoras: El resultado del MRPP mostró que sólo había diferencias significativas en la composición de especies entre los árboles de Clusia 
y los de Heliocarpus americanus, Meriania y Saurauia brachybotrys (Cuadro 3). Para los grupos, sólo hubo diferencias significativas entre el grupo 1 y 2, mientras que entre los grupos 2 y 3 no hubo diferencias (Cuadro 3 ). Hay que indicar que los grupos 1 y 3 presentaron diferencias, aunque no alcanzaron a ser significativas.

El resultado del NMS muestra que algunos forófitos de la misma especie se agrupan en diferentes regiones del gráfico de la dimensión 1 vs. dimensión 2 del análisis (Fig. 6). En el lado izquierdo se agruparon árboles de Schefflera ferruginea. Los árboles de Saurauia brachybotrys estuvieron más dispersos en el gráfico. En la parte superior se agruparon forófitos de Clusia sp. mientras que en la parte inferior los de Heliocarpus americanus.

El análisis de correlación de Spearman para las dimensiones 1 y 2 del NMS, y los parámetros microambientales medidos, sugieren que los factores intensidad de luz y temperatura estuvieron relacionados positivamente con la dimensión 1, y los parámetros DAP y temperatura estaban relacionados negativamente con la dimensión 2 (Cuadro 4). Los parámetros $\mathrm{pH}$ y humedad, no estuvieron correlacionados con las dimensiones del NMS. Así entonces, se tiene que los forófitos que se encuentra en el lado izquierdo (Fig. 6) recibieron baja intensidad de luz y presentaron bajas temperaturas, en comparación con los árboles del lado derecho. De igual forma, los árboles de la parte superior presentaron un DAP y una temperatura baja frente a los árboles de la parte inferior del gráfico (Fig. 6). Los resultados indican que los factores DAP, intensidad de luz y temperatura ambiental, afectan la distribución de las comunidades (composición de especies) de líquenes en la zona de estudio. La riqueza de especies y la diversidad por forófito no estuvieron correlacionadas con las variables microambientales.

El resultado del análisis de especies indicadoras para las cinco especies de forófitos mostró que solamente tres especies de líquenes presentaron preferencias significativas $(\mathrm{p}<0.05)$ a ciertos tipos de forófito (Cuadro 5). $\mathrm{La}$ especies con preferencia por el forófito
CUADRO 3

MRPP para todas las comparaciones

TABLE 3

MRPP for all comparisons

\begin{tabular}{lcc}
\multicolumn{1}{c}{ Comparación } & $\mathrm{T}$ & $\mathrm{p}$ \\
Clusia vs. Heliocarpus & -2.67 & 0.011 \\
Clusia vs. Meriania & -3.09 & 0.007 \\
Clusia vs. Saurauia & -2.86 & 0.007 \\
Clusia vs. Schefflera & -1.65 & 0.061 \\
Heliocarpus vs. Meriania & -0.92 & 0.175 \\
Heliocarpus vs. Saurauia & -0.28 & 0.381 \\
Heliocarpus vs. Schefflera & -1.65 & 0.054 \\
Meriania vs. Saurauia & -1.35 & 0.095 \\
Meriania vs. Schefflera & -1.46 & 0.076 \\
Saurauia vs. Schefflera & 0.29 & 0.539 \\
Grupo 1 vs.Grupo 2 & -1.85 & 0.040 \\
Grupo 1 vs.Grupo 3 & -1.57 & 0.071 \\
Grupo 2 vs.Grupo 3 & -0.69 & 0.222 \\
\hline
\end{tabular}

$\mathrm{T}$ es el estadístico de prueba para el MRPP y p la probabilidad asociada a este.

$\mathrm{T}$ is the test statistic for the MRPP and $\mathrm{p}$ its associated probability.

\section{CUADRO 4}

Correlación de Spearman para las dos dimensiones del NMS, los parámetros microclimáticos y de sustrato, y la riqueza de especies de líquenes

TABLE 4

Spearman correlation for the two dimensions of the NMS, microclimate and substrate parameters, and lichen species richness

\begin{tabular}{lccc}
\multicolumn{1}{c}{$\mathrm{r}(\mathrm{p})$} & DIM1 & DIM2 & $\mathrm{S}$ \\
$\mathrm{S}$ & $0.25(0.23)$ & $0.26(0.21)$ & 0 \\
$\mathrm{pH}$ & $0.31(0.14)$ & $-0.087(0.68)$ & $0.021(0.92)$ \\
$\mathrm{DAP}$ & $0.16(0.45)$ & $-0.42(0.038)$ & $-0.29(0.16)$ \\
Intensidad luz & $0.54(0.0057)$ & $-0.22(0.29)$ & $0.13(0.53)$ \\
Humedad relativa & $0.31(0.13)$ & $0.024(0.91)$ & $0.29(0.16)$ \\
Temperatura & $-0.49(0.013)$ & $-0.44(0.029)$ & $-0.11(0.61)$ \\
Número de lenticelas & $-0.002(0.99)$ & $-0.34(0.10)$ & $-0.27(0.20)$ \\
Tamaño de las lenticelas & $-0.25(0.23)$ & $0.062(0.77)$ & $-0.028(0.89)$ \\
\hline
\end{tabular}

fueron: Arthonia microsperma (hacia Meriania), Cladonia ceratophylla y sorediado 8 (hacia Clusia). De igual forma, para los tres grupos formados a lo largo del camino, únicamente siete especies de líquenes mostraron 


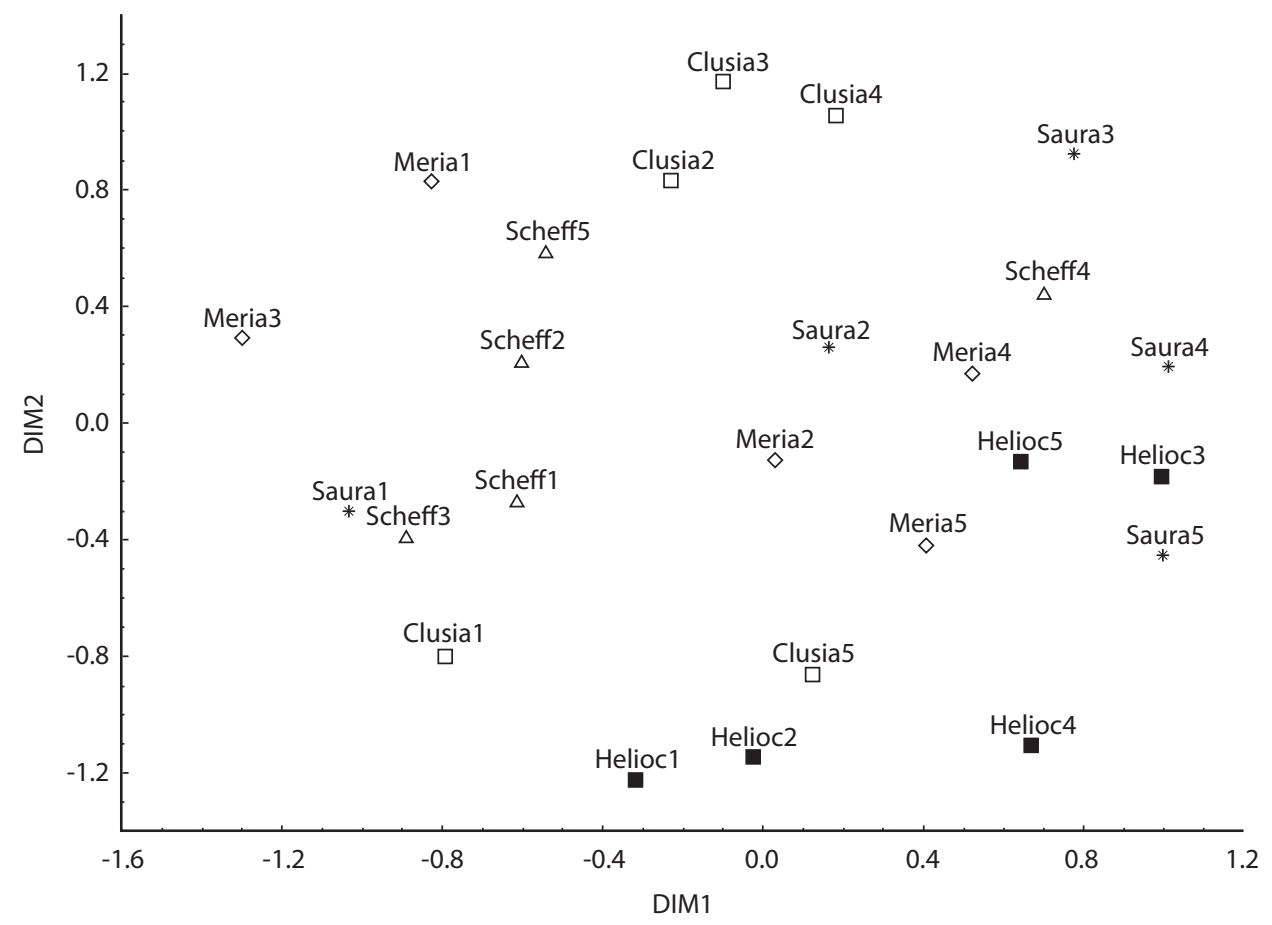

Fig. 6. Dispersión para las dos primeras dimensiones del NMS para los 25 forófitos. Fig. 6. Dispersion for the two first dimensions of the NMS for the 25 phorophytes.

\section{CUADRO 5}

Especies indicadoras para los cinco forófitos y los tres grupos

TABLE 5

Indicator species for the five phorophytes and the three groups

\begin{tabular}{lccccc}
\multicolumn{1}{c}{ Especie } & Grupo o forófito & VIO & VIE & SD & $p$ \\
Cladonia ceratophylla & Clusia & 42.9 & 20.1 & 9.81 & $0.0474^{*}$ \\
Arthonia aff. microsperma & Meriania & 55.0 & 19.6 & 11.02 & $0.0218^{*}$ \\
Sorediado 8 & Clusia & 80.0 & 25.1 & 12.03 & $0.0014^{*}$ \\
Arthonia aff. microsperma & 2 & 30.6 & 17.6 & 8.74 & $0.0966^{\mathrm{b}}$ \\
Cladonia ceratophylla & 1 & 32.7 & 18.8 & 8.57 & $0.0836^{\mathrm{b}}$ \\
Herpothallon sp.6 & 1 & 28.6 & 15.6 & 5.90 & $0.0742^{\mathrm{b}}$ \\
Sorediado 7 & 2 & 40.1 & 25.5 & 9.38 & $0.0704^{\mathrm{b}}$ \\
Ocellularia clandestinal & 1 & 28.6 & 13.0 & 7.49 & $0.0698^{\mathrm{b}}$ \\
Graphis adpressa & 1 & 28.6 & 12.2 & 7.81 & $0.0644^{\mathrm{b}}$ \\
Phaeographis brasiliensis & 1 & 28.6 & 14.7 & 6.18 & $0.0644^{\mathrm{b}}$ \\
Thelotrema leucomelanum & 1 & 40.1 & 18.8 & 9.10 & $0.0462^{*}$ \\
Sorediado 8 & 1 & 42.6 & 20.4 & 9.47 & $0.0338^{*}$ \\
\hline
\end{tabular}

* Significativamente indicadora y $\mathrm{b}$ marginalmente indicadora.Valor indicador observado (IVO) y esperado (IVE) y desviación estándar (SD). Indicator value observed (IVO) and expected (IVE) and standard deviation (SD). 
preferencias marginales $(\mathrm{p}<0.1)$ por ciertos forófitos y para dos fueron significativas (Cuadro 5). Las especies Arthonia microsperma (grupo 2), Cladonia ceratophylla (grupo 1), sorediado 7 (grupo 2), Graphis adpressa (grupo 1), Herpothallon rubrocinctum (grupo 1), Ocellularia clandestina (grupo 1) y Phaeographis brasiliensis (grupo 1) presentaron preferencias marginales para los grupos señalados en paréntesis, mientras que sorediado 8 y Thelotrema leucomelanum mostraron preferencias significativas por el grupo 1 (Cuadro 5).

\section{DISCUSIÓN}

La diversidad alfa fue en promedio de 5.4 especies de líquenes por forófito, lo cual es bajo comparado con el estudio de Cáceres et al. (2007) en Brasil (8.6 especies) y con el de Nöske (2004) (25 especies) en Ecuador, aunque hay que aclarar que el tamaño de la unidad de muestreo fue más amplio para estos lugares. La diversidad beta fue alta comparada con otros estudios (Wolf 1993, Nöske 2004). Así entonces, se tiene que en la finca Zíngara la diversidad beta contribuyó más a la diversidad general que la diversidad alfa. Los resultados encontrados concuerdan con lo reportado por Cáceres et al. (2007) en Brasil y contrastan con estudio de Nöske (2004) en Ecuador, en el que la diversidad alfa tuvo la mayor contribución. La alta diversidad beta puede ser explicada por el método de muestreo empleado, el cual hace énfasis en recolectar microlíquenes e identificarlos hasta el nivel de especie, con lo cual se encuentran muchas especies raras, inconspicuas, crípticas y estériles, y aumentan de esta forma la heterogeneidad entre muestras (Komposch \& Hafellner 2000, Cáceres et al. 2007). Además, en los bosques montanos es difícil detectar los microlíquenes por la alta cobertura de otros epífitos, por lo cual se incrementa el número de especies raras. De esta forma, estos resultados contribuyen a la idea de que en los trópicos el componente más importante en la diversidad total de los microlíquenes es la diversidad beta (Montfoort \& Ek 1990, Wolf
1993, Komposch \& Hafellner 2000, Cáceres et al. 2007).

Otra explicación al número alto de especies raras es la alta heterogeneidad ambiental dentro de los bosques húmedos tropicales, lo cual genera una alta disponibilidad de microhábitats (Gradstein et al. 1996). De esta forma, es de esperar que la distribución de los líquenes esté restringida a algunas zonas del bosque (distribución dispersa) debido a que muchas especies crecen bajo condiciones de micrositio específicas, lo cual conduce a una diversidad beta alta (Sipman \& Harris 1989, Rivas-Plata et al. 2008). Este patrón de distribución de los líquenes también explica la baja eficiencia del muestreo que evidencia la curva de acumulación de especies, pues se requeriría muestrear un alto número de árboles en diferentes microhábitats para encontrar el mayor número de especies de líquenes (Gradstein et al. 1996, Rivas-Plata et al. 2008)

Ahora bien, al tener en cuenta el resultado del MRPP y del NMS, y los parámetros microclimáticos y de sustrato, es probable que las diferencias en la composición de especies encontradas sean producto de un gradiente ambiental (principalmente DAP e intensidad de luz). Esto coincide con los resultados de estudios en la cordillera central de Colombia (Wolf 1993) y en un bosque montano en Costa Rica (Holz 2003), donde los factores luz, humedad relativa, $\mathrm{pH}$ y estructura de la corteza afectaron la formación de comunidades de líquenes. Lo anterior, también, se evidencia en el análisis que se hizo para los tres grupos, pues las tres zonas presentaron diferencias microambientales y en la composición de especies de líquenes lo cual es consecuencia de una estructura vegetal diferente. El alto solapamiento en la composición de especies entre los grupos 2 y 3 se debe probablemente a que presentan una cobertura vegetal muy semejante, por lo cual las condiciones climáticas (luz y humedad relativa) no varían demasiado entre las dos zonas (Sipman \& Harris 1989, Rivas-Plata et al. 2008). Similares resultados han sido reportados por otros autores, quienes han observado que las variables irradianza, humedad relativa, contenido de 
nutrientes del sustrato y estructura de la corteza, afectan la distribución de las comunidades de líquenes en los árboles (Cornelissen \& ter Steege 1989, Wolf 1993, Holz 2003).

La diversidad alfa no estuvo relacionada significativamente con ninguna de las variables estudiadas. Este último resultado se debe probablemente a que en los bosques montanos las comunidades de líquenes, además de estar influenciadas por los factores ambientales, interactúan con briófitos, helechos y angiospermas, los cuales influencian la riqueza y la composición de las comunidades liquénicas junto con las condiciones de microhábitat (Kelly et al. 2004). Otra posible explicación, es que en este estudio no se consideraron otros factores como la capa de humus que cubre la corteza y el efecto estocástico en la dispersión de las especies de líquenes, los cuales podrían afectar su riqueza (Kelly et al. 2004, Cáceres et al. 2007).

Con base en los resultados del MRPP, NMS, análisis de la diversidad beta y de especies indicadoras, se puede decir que no hay una relación específica entre la especie de liquen y la del forófito, sino preferencias hacia características climáticas y del sustrato (Sipman \& Harris 1989, Cáceres et al. 2007). Esto puede deberse a que la alta diversidad vegetal presente en el trópico podría obscurecer tales preferencias de forófito, y en lugar de adicionar, mitigar la variación en las características de la corteza, lo cual causa una diferenciación menos obvia entre árboles. Se puede esperar en los bosques tropicales patrones menos evidentes de líquenes con preferencia de forófito que los reportados para los bosques templados (Cáceres et al. 2007). También se asume que las preferencias de forófito reducen la probabilidad de establecimiento exitoso de un liquen, en donde se considera que la dispersión de las diásporas es en gran parte estocástica y la alta diversidad de árboles reduce la abundancia de sustratos adecuados (Cáceres et al. 2007).

En conclusión, no se observó especificidad de forófito de los líquenes cortícolas, sino que las condiciones microclimáticas y de sustrato influenciaron la composición de especies de líquenes, principalmente la intensidad de luz y el DAP. Además, no se encontró relación entre la riqueza y las variables de microambientales, lo cual se puede deber a que en los bosques montanos riqueza de líquenes interactúa con las con condiciones microclimáticas y con los epífitos.

\section{AGRADECIMIENTOS}

A Manuel y Jorge Giraldo por permitir colectar líquenes en la Finca Zíngara. Al laboratorio de biología de la Universidad del Valle por el préstamo de los equipos y de los laboratorios para la realización de este trabajo.

\section{RESUMEN}

El objetivo de este trabajo fue evaluar la especificidad de forófito y las preferencias microambientales de los líquenes cortícolas en el Bosque de la Finca Zíngara (Cali, Colombia). Asimismo, se seleccionaron cinco individuos de cinco especies de árboles. Además, se identificaron las especies de líquenes presentes en un cuadrante de $0.50 \times 0.20 \mathrm{~m}^{2}$ ubicado en el tronco de cada árbol a $1.3 \mathrm{~m} \mathrm{de}$ altura. También, se midieron parámetros microambientales como $\mathrm{pH}$ de la corteza, diámetro a la altura del pecho (DAP), estructura de la corteza, humedad relativa e irradianza. Para detectar las preferencias de forófito, se realizó un escalamiento no métrico multidimensional (NMS) y un análisis de especies indicadoras. Luego se efectúo un análisis de correlación de Spearman para evaluar la relación entre las variables ambientales y los agrupamientos encontrados en el NMS. Se encontraron 69 especies de líquenes, de los cuales 37 fueron determinados hasta especie, 18 hasta género y 14 no fueron determinados. El resultado del NMS mostró que algunos individuos de la misma especie de árbol se agruparon a lo largo de las dimensiones del análisis, y están relacionados con los factores intensidad de luz, temperatura y DAP. Sólo tres especies mostraron preferencia por ciertas especies de árboles (Arthonia microsperma por Meriania sp., Cladonia ceratophylla y sorediado 8 por Clusia sp.), lo cual sugiere ausencia de preferencias de forófito.

Palabras clave: líquenes cortícolas, especificidad de forófito, parámetros microambientales, escalamiento no métrico multidimensional (NMS), correlación, análisis de especies indicadoras. 


\section{REFERENCIAS}

Aragón, G., I. Martínez, P. Izquierdo, R. Belinchón \& A. Escudero. 2010. Effects on forest Management on epiphytic lichen diversity in Mediterranean forests. Appl.Veg. Sci. 13: 183-194.

Armenteras, D., V.C. Cadena \& R.P. Moreno. 2007. Evaluación del estado de los bosques de niebla y de la meta 2010 en Colombia. Instituto de Investigación de Recursos Biológicos Alexander von Humboldt, Bogotá, D.C., Colombia.

Barillas, R., R. Lücking \& S. Winkler. 1993. Vergesellschaftungen foliikoler Flechten im Biotopo del Quetzal, Guatemala. Cryptogamie Bryol. L. 14: 49-68.

Belinchón, R., I. Martínez, M.A. Otálora, G. Aragón, J. Dimas \& A. Escudero. 2009. Fragment quality and matrix affect epiphytic performance in a Mediterranean forest landscape. Am. J. Bot. 96: 1974-1982.

Boonpragob, K. \& W. Polyiam. 2007. Ecological groups of lichens along environmental gradients on two different host tree species in the tropical rain forest at Khao Yai National Park, Thailand. Bibl. Lichenol. 96: 25-48.

Cáceres, M.S., R. Lücking \& G. Rambold. 2007. Phorophyte specificity and environmental parameters versus stochasticity as determinants for species composition of corticolous crustose lichen communities in the Atlantic rain forest of northeastern Brazil Mycol. Prog. 10: 190-210.

Coppins, B.J. \& P. Wolseley. 2002. Lichens of tropical forests, p. 113-131. In R. Watling, J.C. Frankland, A.M. Ainsworth, S. Isaac \& C.H. Robinson (eds.). Tropical Mycology: Volume 2, Micromycetes. CABI, Wallingford, EEUU.

Cornelissen, J.H. \& H. ter Steege. 1989. Distribution and ecology of epiphytic bryophytes and lichens in dry evergreen forest of Guyana. J. Trop. Ecol. 5: 131-150.

Giraldo, J. 1988. Estudio fenológico de una comunidad vegetal en un bosque montano húmedo en la cordillera occidental. Tesis de pregrado, Universidad del Valle, Cali, Colombia.

Gentry, A.H. \& C.H. Dodson. 1987. Diversity and biogeography of neotropical vascular epiphytes. Ann. Mo. Bot. Gard. 74: 205-233

Gradstein, S.R. 1992. The vanishing tropical rain forest as an environment for bryophytes and lichens, p. 234258. In J.W. Bates \& A.M. Farmer (eds.). Bryophytes and lichens in a changing environment. Clarendon, Oxford, Inglaterra.
Gradstein, S.R., P. Hietz, R. Lücking, A. Lücking, H.J.M. Sipman, H.F.M. Vester, J.H.D. Wolf \& E. Gardette. 1996. How to sample the epiphytic diversity of tropical rain forests. Ecotropica 2: 59-72.

Hauck, M. \& M. Spribille. 2005. The significance of precipitation and substrate chemistry for epiphytic lichen diversity in spruce-fir forests of the Salish Mountains, northwestern Montana. Flora 200: 547-562

Holz, I. 2003. Diversity and ecology bryophytes and macrolichens in primary and secondary montane Quercus forests, Cordillera de Talamanca, Costa Rica. PhD Thesis, University of Göttingen, Göttingen, Alemania

Holz, I. \& S.R. Gradstein. 2005. Cryptogamic epiphytes in primary and recovering upper montane oak forests of Costa Rica- species richness, community composition and ecology. Plant. Ecol. 178: 89-109.

Kelly, D.L., G. O’Donovan, J. Feehan, S. Murphy, S.O. Drangeid \& L. Marcano-Berti. 2004. The epiphyte communities of a montane rain forest in the Andes of Venezuela: patterns in the distribution of the flora. J. Trop. Ecol. 20: 643-666.

Komposch, H. \& J. Hafellner. 1999. List of lichenized fungi so far observed in the tropical lowland rain forest plot Surumoni (Venezuela, Estado Amazonas). Fritschiana 19: 1-10.

Komposch, H. \& J. Hafellner. 2000. Diversity and vertical distribution of lichens in a Venezuelan tropical lowland rain forest. Selbyana 21: 11-24.

Komposch, H. \& J. Hafellner. 2002. Life form diversity of lichenized fungi in an Amazon lowland rainforest. Bibl. Lichenol. 82: 311-326.

Komposch, H. \& J. Hafellner. 2003. Species composition of lichen dominated corticolous communities: a lowland rain forest canopy compared to an adjacent shrubland in Venezuela. Bibl. Lichenol. 86: 351-367.

Lücking, R. 1999. Ecology of foliicolous lichens at the "Botarrama" Trail (Costa Rica), a neotropical rain forest. I. Species composition and its ecogeographical implications. Biotropica 31: 553-56.

Lücking, R., E. Rivas-Plata, J.L. Chaves, L. Umaña \& H.J. Sipman. 2009. How many tropical lichens are there... really?, p. 399-417. In A. Thell, M.R.D. Seaward \& T. Feuerer (eds.). Diversity of Lichenology - Anniversary Volume. Bibl. Lichenol, Berlin-Stuttgart, Alemania.

Marcelli, M.P. 1992. Ecologia Liquênica nos Manguezais do Sul-Sudeste. Bibl. Lichenol. 47: 1-310. 
McCune, B. 2000. Lichen Communities as Indicators of Forest Health. Bryologist 103: 353-356.

McCune, B., J. Dey, J. Peck, D. Cassell, K. Heiman, S. Will-Wolf \& P. Neitlich. 1997. Repeatability of community data: species richness versus gradient scores in large-scale lichen studies. Bryologist 100: 40-46.

McCune, B., J.B. Grace \& D.L. Urban. 2002. Analysis of ecological communities. MjM Software, Gleneden Beach, Oregon.

Merwin, M.C. \& N.M. Nadkarni. 2001. 100 years of tropical bryophyte and lichen ecology: a bibliographic guide to the literature from 1901-2000. Trop. Bryol. 21: $97-118$

Montfoort, D. \& R. Ek. 1990. Vertical distribution and ecology of epiphytic bryophytes and lichens in a lowland rain forest in Utrecht, French Guiana. Institute of Systematic Botany, Utrecht, Holanda.

Nash, T.H. 1996. Lichen biology. Cambridge University, Cambridge, Inglaterra.

Nash, T.H. \& A. Olafsen. 1995. Climate change and the ecophysiological response of Arctic lichens. Lichenologist 27: 559-565.

Nöske, N. 2004. Effekte anthropogener Störung auf die Diversität Cryptogamischer Epiphyten (Flechten, Moose) in einem Bergregenwald in Südecuador. Ph.D. Thesis, Mathematisch-Naturwissenschaftliche Fakultät, Georg-August-Universität zu Göttingen, Alemania.

Nöske, N.M. \& H.J. Sipman. 2004. Cryptogams of the Reserva Biológica San Francisco (Province ZamoraChinchipe, Southern Ecuador) II. Lichens. Cryptogamie Mycol. 25: 91-100.

Nowak, R. \& S. Winkler. 1975. Foliicolous lichens of Choco, Colombia, and their substrate abundances. Lichenologist 7: 53-58.

Renhorn, K.E., P.A. Esseen, K. Palmqvist \& B. Sundberg. 1997. Growth and vitality of epiphytic lichens. I. Responses to microclimate along a forest edge-interior gradient. Oecologia 109: 1-9.
Rivas-Plata, E., R. Lücking, A. Aptroot, H.J.M. Sipman, J.L. Chaves, L. Umaña \& D. Lizano. 2006. A first assessment of the Ticolichen biodiversity inventory in Costa Rica: the genus Coenogonium (Ostropales: Coenogoniaceae), with a world-wide key and checklist and a phenotype based cladistic analysis. Fungal Divers. 1: 1-67.

Rivas-Plata, E., R. Lücking \& H.T. Lumbsch. 2008. When family matters: an analysis of Thelotremataceae (Lichenized Ascomycota: Ostropales) as bioindicators of ecological continuity in tropical forests. Biodivers. Conserv. 17: 1319-1351.

Rosabal, D., A.R. Burgaz \& R. de la Masa. 2010. Diversity and distribution of epiphytic macroliches on tree trunks in two slopes of the montane rainforest of Gran Piedra, Santiago de Cuba. Bryologist 113: 313-321.

Selva, S.B. 1996. Using lichens to assess ecological continuity in northeastern forests, p. 35-48. In M. Byrd (ed.). Eastern old-growth forests - prospects for rediscovery and recovery. Island, Washington, D.C., EEUU.

Sipman, H.J. \& R.C. Harris. 1989. Lichens, p. 303-309. In H. Lieth \& M.J.A. Werger (eds.). Tropical rain forest ecosystems. Elsevier Science, Amsterdam, Holanda.

Sipman, H.J. 2006. Lichen determination keys available on internet, Botanischer Garten Und Botanisches Museum Berlin-DahleM. (Consultado: 14 Noviembre 2009, http://www.bgbm.org/Sipman/keys/default. htm).

Statsoft. 2004. STATISTICA 7.0. (Consultado: 3 Julio 2009, http://www.statsoft.com.au/v7.htm).

Werth, S., H. Tommervik \& A. Elvebakk. 2005. Epiphytic macrolichen communities along regional gradients in northern Norway. J. Veg. Sci.16: 199-208.

Wolf, J.H. 1993. Diversity patterns and biomass of epiphytic bryophytes and lichens along an altitudinal gradient in the northern Andes. Ann. Mo. Bot. Gard. 80: 928-960. 